\title{
MUSTELA VISON ACE2 FUNCTIONS AS A RECEPTOR FOR SARS-CORONAVIRUS
}

\author{
Lindsay K. Heller, Laura Gillim-Ross, Emily R. Olivieri, and
}

David E. Wentworth*

\section{INTRODUCTION}

Coronaviruses (CoVs), members of the order Nidovirales, are enveloped, positivesense, single-stranded RNA viruses. ${ }^{1}$ The genomes of CoVs are among the largest of known RNA viruses, and range in size from 27 to 32 kilobases in length. ${ }^{1}$ During replication within the host cell, all Nidovirales produce a set of 3' nested transcripts that share a short leader sequence at the 5 ' terminus. ${ }^{1} \mathrm{CoVs}$ are known for their crown-like appearance, which is due to the spike (S) glycoproteins projecting from the surface of the virion. Interaction of $S$ and cellular receptors facilitates entry of the virus, and this interaction is a principal factor in the tissue tropism and the species specificity of CoVs.

In 2002-2003, there was an outbreak of severe acute respiratory syndrome (SARS) in Guangdong Province, China. This outbreak subsequently affected 29 countries and resulted in 8,096 cases of SARS, of which 774 were fatal. ${ }^{2}$ The causative agent was determined to be a previously unrecognized $\mathrm{CoV}$, which became known as SARS-CoV. The SARS-CoV outbreak likely resulted from zoonotic transmission. Although SARS$\mathrm{CoV}$ was isolated from several species, the natural reservoir of its progenitor remains to be discovered. Of the animals examined to date, SARS-CoV was most frequently isolated from Himalayan palm civets, which are sold in live animal markets in Guangdong and in other regions of Southeast Asia. ${ }^{3}$ The role palm civets played in the SARS outbreak is unclear. However, SARS-CoV-like viruses isolated from palm civets appeared to be under strong selective pressure and are genetically most similar to viruses infecting humans early in the outbreak. ${ }^{4}$ The selective pressure of replication in palm civet may have generated strains that could be more easily transmitted to humans. Palm civets are carnivores in the suborder Fissipedia. Other Fissipedia include raccoon dog, dog, cat, raccoon, skunk, ferret (Mustela putorius), and mink (Mustela vison). SARS-CoV-like virus was also detected in a raccoon dog in a live animal market. ${ }^{3}$ Furthermore, Fissipedia

\footnotetext{
* Lindsay Heller, Laura Gillim-Ross, New York State Department of Health, Albany, New York 12002. Emily Olivieri, David E. Wentworth, New York State Department of Health, and State University of New York, Albany, New York 12002.
} 
such as cat, ferret, ${ }^{5}$ and palm civet $^{6}$ have been experimentally infected with SARS$\mathrm{CoV} /$ Urbani.

Previous analysis of cell lines and primary cells derived from diverse species led to the discovery that a mink lung cell line $(\mathrm{Mv} 1 \mathrm{Lu})$ was permissive to SARS-CoV. ${ }^{7}, 8$ Angiotensin-converting enzyme $2(\mathrm{ACE} 2)^{9}$ and CD209L ${ }^{10}$ are host cell-surface proteins that function as receptors for SARS-CoV. However, human ACE2 (hACE2) is a more efficient receptor than is CD209L, in vitro. Therefore, we set out to determine whether ACE2 from M. vison (mvACE2) is a functional receptor for SARS-CoV. Our data shows ACE2 RNA was expressed by Mv1Lu cells. We subsequently sequenced, cloned, and expressed mvACE2 in nonpermissive cells, to determine its SARS-CoV receptor activity. Human, palm civet, rat, mouse, chicken, and mink ACE2 were compared to identify differences that are important in species specificity and to discern regions within ACE2 that may impact its function as a SARS-CoV receptor.

\section{RESULTS AND DISCUSSION}

Expression of ACE2 RNA in SARS-CoV susceptible human (Huh7, HEK293T), African green monkey (VeroE6), and Mv1Lu cell lines ${ }^{7}$ was analyzed by RT-PCR (chapter 4.8 this volume). The amount of ACE2 amplicon differed among the various cell lines. VeroE6 had the strongest ACE2 amplicon, whereas Mv1Lu cells showed the weakest RT-PCR product. However, we subsequently found that the human/mouse consensus primers used initially were not $100 \%$ conserved with the mvACE2 nucleotide sequence. RT-PCR amplification of ACE2 from VeroE6, Huh7, and Mv1Lu cells with ACE2-1446 (GGAACTCTACCATTTACTTACA) and ACE2-1991R (TCCAAGAGCTGATTTTAGGCTTAT), which both have $100 \%$ identity with mvACE2, showed a robust amplicon from Mv1Lu total RNA (Figure 1). Glyceraldehyde-3phosphate dehydrogenase (G3PDH) was also amplified, as a control for RNA concentration and integrity (Figure 1$).^{7}$ The complete open reading frame of human ACE2 (hACE2) or mvACE2 was amplified from RNA isolated from the Huh7 or Mv1Lu cell line, respectively. The consensus sequences of human and mink ACE2 open reading frames were determined by direct sequencing of RT-PCR amplicons. Amplicons of hACE2 and mvACE2 open reading frames were cloned and confirmed by sequence analysis. The deduced amino-acid sequence identity/similarity of the open reading frames of human, palm civet, mink, mouse, rat, and chicken ACE2 were compared using VectorNTI Advance 9.1 (Table 1). The data demonstrate that mink and palm civet ACE2
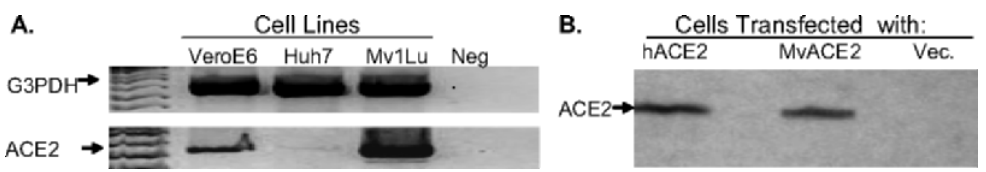

Figure 1. A. ACE2 transcript identified in M. vison lung epithelial cells. G3PDH and ACE2 RT-PCR amplicons from $1 \mu \mathrm{g}$ of total RNA were visualized by ethidium bromide staining after agarose gel electrophoresis. A negative control that lacks RNA template (Neg) was also included. B. Expression of ACE2 Protein. HEK-293T cells were transfected with human (hACE2), mink (MvACE2), or the pIRES2-EGFP (BD-Biosciences) expression vector alone (Vec.), and lysates were harvested at $48 \mathrm{~h}$ post-transfection. Immunoblotting of cell lysates was performed with polyclonal antibody against human ACE2 (R\&D systems). 
Table 1. Percent amino-acid identity/similarity of ACE2 orthologs.

$\begin{array}{llllllll}\text { Species } & \text { Human } & \text { P. Civet } & \text { Mink } & \text { Mouse } & \text { Rat } & & \text { Chicken } \\ \text { Human } & 100 / 100 & & & & & \\ \text { P. Civet } & 83 / 87 & 100 / 100 & & & & \\ \text { Mink } & 83 / 87 & 88 / 90 & 100 / 100 & & & \\ \text { Mouse } & 82 / 87 & 82 / 86 & 81 / 87 & 100 / 100 & & \\ \text { Rat } & 83 / 87 & 81 / 85 & 80 / 85 & 90 / 93 & 100 / 100 & \\ \text { Chicken } & 62 / 71 & 63 / 72 & 62 / 71 & 61 / 71 & 61 / 71 & 100 / 100\end{array}$

have the greatest amino-acid identity/similarity (88/90\%), and additional analysis indicated that mink and palm civet ACE2 have common substitutions, when compared to hACE2.

To investigate the receptor activity of mvACE2, we generated eukaryotic expression constructs of hACE2 and mvACE2 using pIRES2-EGFP. Immunoblot showed that both hACE2 and mvACE2 constructs expressed protein when they were transfected into HEK$293 \mathrm{~T}$ cells (Figure 1B). To test whether mvACE2 could function as a SARS-CoV receptor, normally nonpermissive BHK-21 cells were transiently transfected with hACE2, mvACE2, or empty pIRES2-EGFP expression plasmids, and then analyzed for susceptibility to SARS-CoV (Figure 2). To identify SARS-CoV entry, we isolated total RNA at $1 \mathrm{~h}$ or $24 \mathrm{~h}$ post-inoculation and analyzed it using our multiplex RT-PCR assay, which amplifies G3PDH, SARS-CoV genomic RNA (gRNA), and SARS-CoV subgenomic RNA (sgRNA) ${ }^{7}$; the latter is indicative of virus entry. SARS-CoV sgRNA was amplified in both hACE2 and mvACE2 transfected cells at $24 \mathrm{~h}$ post-inoculation. In contrast, control cells transfected with pIRES2-EGFP, or that were mock-transfected, showed amplification of G3PDH and some gRNA, presumably from residual input virus (Figure 2). These data show that mvACE2 is a functional receptor for SARS-CoV.

We used the crystal structure of hACE2 ${ }^{11}$ to map amino acid differences between hACE2 and mvACE2. Many of the amino-acid substitutions localize to the surface of the protein, and some are very close to residues that $\mathrm{Li}$ et al. recently showed to be important for binding of a SARS-CoV S1-Ig fusion protein (e.g., K353, D355, R357). ${ }^{12}$ We identified differences at residues H34, D38, N61, L79, N103, Q305, Q325, E329, and G354 of hACE2 that may also be important SARS-CoV S-ACE2 interaction. Given the strong effect of $\mathrm{K} 353 \mathrm{H}$ substitution on $\mathrm{S} 1-\mathrm{Ig}$ binding to hACE2, ${ }^{12}$ the G354Q substitution that we identified in mvACE2 is also likely to decrease SARS-CoV/Urbani binding. We have passaged SARS-CoV/Urbani in mvACE2-expressing Mv1Lu cells, and selected variants that replicate to higher titer and form larger plaques than wild-type virus (chapter 4.8 this volume). Our analysis of the $\mathrm{S}$ gene from these variants is likely to identify mutations that compensate for G354Q in mvACE2. ACE2 amino-acid alignment of good and poor SARS-CoV/Urbani receptors suggests that additional residues or post-translational modifications of ACE2 affect SARS-CoV entry (Olivieri et al., this volume).

Our data demonstrate that mvACE2 RNA is expressed by SARS-CoV susceptible Mv1Lu cells, that it is closely related to palm civet ACE2, and that mvACE2 is a functional receptor for SARS-CoV. We also identified species-specific amino-acid variations within ACE2 that are likely to influence its SARS-CoV receptor activity. Additionally, our results strongly suggest that, like palm civet, raccoon, dog, cat, and ferret, ${ }^{3,5,6}$ mink may be another member of the suborder Fissipedia that is susceptible to SARS-CoV. Therefore, mink may not only provide an additional animal model for 


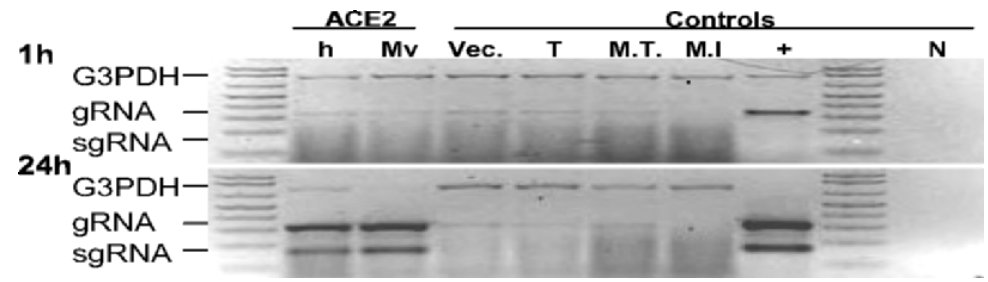

Figure 2. Transfection of MvACE2 confers susceptibility to SARS-CoV. Normally nonpermissive BHK-21 cells were transfected with hACE2 (h), mvACE2 (Mv), empty expression plasmid pIRES2-EGFP (Vec.), pEGFP-N1 (T) to monitor transfection efficiency, or were mock-transfected (M.T.). Cells were inoculated with SARS-CoV (MOI of $\sim 0.01$ ) at $48 \mathrm{~h}$ post-transfection. G3PDH, SARS-CoV gRNA and sgRNA were amplified from $1 \mu \mathrm{g}$ of total RNA isolated at $1 \mathrm{~h}$, or $24 \mathrm{~h}$ post-inoculation. Mock-inoculated BHK-21 (M.I.) and SARS$\mathrm{CoV}$ inoculated VeroE6 (+) were also included as controls. N is dH2O template RT-PCR control. Amplicons were visualized by ethidium bromide staining after electrophoresis, and the images have been contrast inverted.

SARS-CoV pathogenesis studies, but they may also have potential as a North American wildlife reservoir of SARS-CoV-like viruses.

We thank Noel Espina for his expert assistance, and the Wadsworth Center Molecular Genetics Core for DNA sequencing. L.G.-R. was supported by an EID fellowship administered by the APHL and funded by the CDC. E.O. was supported by NIH/NIAID training grant T32AI05542901A1. The study was also funded by Diagnostic Hybrids Inc. and by NIH/NIAID grants N01-AI-25490 and P01-AI-0595760.

\section{REFERENCES}

1. K. V. Holmes, in: Fields Virology, vol. 1, edited by D. M. Knipe, P. M. Howley, D. E. Griffin, R. A. Lamb, M. A. Martin, and B. Roizman, (Lippincott Williams \& Wilkins, Philadelphia, 2001), pp. 1187-1203.

2. Summary of probable SARS cases with onset of illness from 1 November 2002 to 31 July 2003, World Health Organization, http://www.who.int/csr/sars/country/table2004_04_21/en/index.html (2004).

3. Y. Guan, B. J. Zheng, Y. Q. He, et al., Isolation and characterization of viruses related to the SARS coronavirus from animals in southern China, Science 302, 276-278 (2003).

4. H. D. Song, C. C. Tu, G. W. Zhang, et al., Cross-host evolution of severe acute respiratory syndrome coronavirus in palm civet and human, Proc. Natl. Acad. Sci. USA 102, 2430-2435 (2005).

5. B. E. E. Martina, B. L. Haagmans, T. Kuiken, et al., SARS virus infection of cats and ferrets, Nature 425, $915(2003)$.

6. D. Wu, C. Tu, C. Xin, et al., Civets are equally susceptible to experimental infection by two different severe acute respiratory syndrome coronavirus isolates, J. Virol. 79, 2620-2625 (2005).

7. L. Gillim-Ross, J. Taylor, D. R. Scholl, et al., Discovery of novel human and animal cells infected by the severe acute respiratory syndrome coronavirus by replication-specific multiplex reverse transcription-PCR, J. Clin. Microbiol. 42, 3196-3206 (2004).

8. E. C. Mossel, C. Huang, K. Narayanan, et al., Exogenous ACE2 expression allows refractory cell lines to support severe acute respiratory syndrome coronavirus replication, J. Virol. 79, 3846-3850 (2005).

9. W. Li, M. J. Moore, N. Vasilieva, et al., Angiotensin-converting enzyme 2 is a functional receptor for the SARS coronavirus, Nature 426, 450-454 (2003).

10. S. A. Jeffers, S. M. Tusell, L. Gillim-Ross, et al., CD209L (L-SIGN) is a receptor for severe acute respiratory syndrome coronavirus, Proc. Natl. Acad. Sci. USA 101, 15748-15753 (2004).

11. P. Towler, B. Staker, S. G. Prasad, et al., ACE2 structures reveal a large hinge-bending motion important for inhibitor binding and catalysis, J. Biol. Chem. 279, 17996-18007 (2004).

12. W. Li, C. Zhang, J. Sui, et al., Receptor and viral determinants of SARS-coronavirus adaptation to human ACE2, EMBO J. 24, 1634-1643 (2005). 